\title{
Study of two methods for comparing laser measuring interferometric systems to reduce the error in the transmission of a unit of length
}

\author{
Irina Novikova ${ }^{*}{ }^{1}$, Denis Sokolov ${ }^{1}$. \\ ${ }^{1}$ All-Union Scientific Research Institute of Physicotechnical and Radiotechnical Measurements, \\ Moscow, Russian Federation, 141570
}

\begin{abstract}
The article presents the results of an experimental study of coaxial and orthogonal methods for comparing laser measuring interferometric systems using a standard measuring complex of length from the State Primary Special Standard of a Unit of Length in the range from 2 to $60 \mathrm{~m}$. transmission errors of the unit of length up to 0.5 microns.
\end{abstract}

\section{Introduction}

The primary special standard of the unit of length (GET 199-2018) is intended for reproduction, storage and transfer of the unit of length - meter [1] to working standards and working measuring instruments. Laser measuring interferometric systems (LMIS), which are part of GET 199-2018, are used to measure displacements of objects in the process of transferring the size of a unit of length to the standard and working measuring instruments, one of such measuring instruments are laser measuring systems (LMS) [2]. To confirm the actual metrological characteristics of the LMS used in the fields of industry and scientific activity, it is necessary to carry out the procedures of their periodic calibrations [3], where the value of the quantity obtained from the LMS is estimated relative to the obtained value of the quantity determined using the LMIS standard. To carry out calibrations at All-Russian Scientific Research Institute for Physical-Engineering and Radiotechnical Metrology "VNIIFTRI" as a standard, a standard measuring complex of length from the composition of GET 199-2018 is used in the length range from 2 to 60 meters (hereinafter referred to as the standard) [4]. Calibration of measuring instruments is carried out taking into account the quantities that affect their metrological characteristics. To ensure the accuracy of measurements, the POWER calibration is carried out in laboratory conditions with the maintenance of temperature indicators from 20 to $25^{\circ} \mathrm{C}$, pressure from 84.0 to $106.7 \mathrm{kPa}$ and humidity not more than $80 \%$. In the process of calibrating the laser measuring system, the value is determined, which is the transmission error of the unit of length and is calculated by the formula (1) [5]:

* Corresponding author: novikova_iv@vniiftri.ru 


$$
\Delta_{\mathrm{ij}}=\mathrm{L}_{\mathrm{ij}}-L_{r . i}
$$

where $\mathrm{L}_{\mathrm{ij}}$ - length measurement result obtained on a LMS;

$\mathrm{L}_{\mathrm{r} . \mathrm{i}}$ - length measurement result obtained on a standard LMIS.

The development of modern industry in the field of production of LMS is associated with the improvement of such technical characteristics as the measurement error. In connection with the reduction of the error of modern LMS, it becomes necessary to improve the methods used to compare the LMS.

At present, when confirming the actual metrological characteristics of the LMS, a coaxial comparison method is used [6], where the studied LMS and the standard are located at the two ends of the reference comparator (base $60 \mathrm{~m}$ long with rails fixed on it for moving the motorized platform) opposite each other (Figure 1). In the coaxial technique, the lengths of the measuring arms of the LMS and the standard change in inverse proportion to each other.

The use of a coaxial technique during measurements ensures the transmission of the size of a unit of length with an error of up to 2.5 microns at a distance in the range of up to $60 \mathrm{~m}$. According to this technique, the optical path lengths of the laser beams of the LMS and the standard differ from each other, which creates a different effect of the unevenness of the medium (fluctuations in temperature, pressure and humidity values) for each laser beam [7]. When using this technique, the unevenness of the medium can significantly affect the measurement error. The use of the coaxial technique in the work increases the time when setting up the equipment, due to the location of the LMS and the standard at a considerable distance from each other $-60 \mathrm{~m}$.

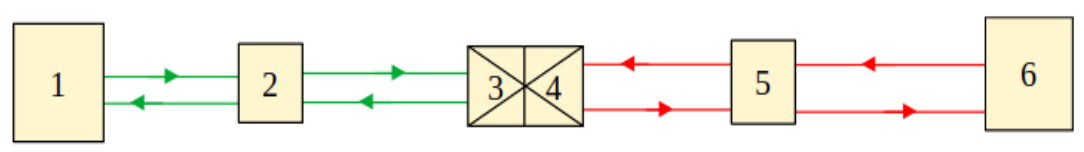

Fig. 1. Scheme of comparison of LMS in a coaxial way. 1 - investigated LMS; 2.5 - node of interference; 3.4 - motorized platform with reflectors; 6 - LMIS (working standard of 2digit length unit); green lines - the direction of radiation of the investigated LMS; red lines direction of radiation of LMIS.

To reduce the error in the transfer of a unit of length from the standard to the LMS, an orthogonal technique is proposed for consideration in which the LMS and the standard are installed at right angles to each other in close proximity (Figure 2). According to this technique, the lengths of the optical paths of the laser radiation of the LMS and the reference will be equal, which will minimize the effect of non-uniformity of the medium on the propagation of radiation and reduce the measurement error. The location of the LMS and the standard according to this technique will reduce the time for setting up the equipment. 


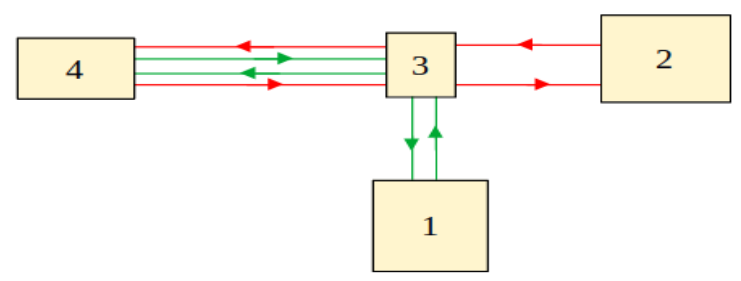

Fig. 2. Scheme of comparison of LMS in the orthogonal way. 1 - LMIS (working standard of 2-digit length unit); 2 - investigated LMS; 3 - node of interference; 4 - motorized platform with a reflector; red lines - the direction of radiation of the investigated LMS; green lines - direction of radiation of LMIS.

\section{Materials and methods}

The article examines the study of the transmission error of the unit of length of the LMS in laboratory conditions, performed simultaneously by coaxial and orthogonal techniques (Figure 3).

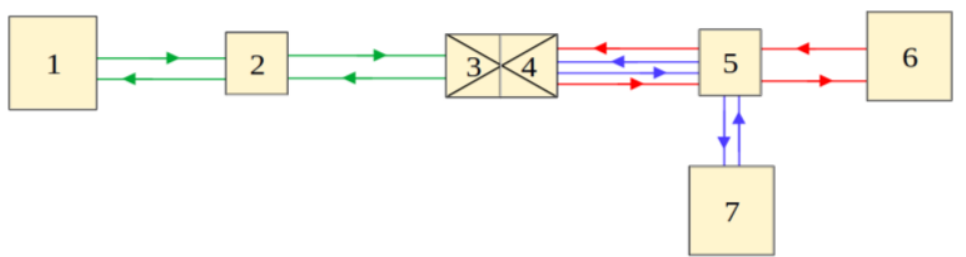

Fig. 3. Scheme for comparing the LMS and the standard by orthogonal and coaxial techniques. 1 - LMS in coaxial technique; 2, 5 - interference node; 3, 4 - motorized platform with reflectors; 6 - standard; 7 - LMS in the orthogonal technique; red lines - the direction of radiation of the standard in the coaxial and orthogonal technique; green lines the direction of the LMS radiation in the coaxial technique; violet lines - the direction of the LMS radiation in the orthogonal technique.

Before carrying out measurements according to the coaxial and orthogonal methods, two LMS were installed on the standard measuring complex at different ends of the measuring base with a length of up to $60 \mathrm{~m}$, where one of the installed LMS was located orthogonally to the standard LMIS in close proximity.

According to the diagram (Figure 3), the motorized platform with reflectors moved from the initial (zero) position to a given distance. The measurement of the length of the segments of the movement of the motorized platform with reflectors was evaluated by each LMS separately and by the LMIS of the standard.

The measurements of the displacements of the motorized platform were carried out simultaneously on two LMS and LMIS standards to ensure uniform measurements. During the study, measurements of a set of lengths in the range of up to 60 meters were carried out by segments, evenly distributed along the measuring basis, at the following control points: 0 , $10,25,30,35,50,60$ meters.

During the time of the measurements, the readings of the meteorological parameters remained within the limits allowed by the operational documentation for the LMS and the 
standard: air temperature $24.47-24.51^{\circ} \mathrm{C}$, air humidity $22.9-23.5 \%$, atmospheric pressure 98.994 - $99.068 \mathrm{kPa}$.

\section{Results and Discussion}

The values of the measured length obtained on the LMS set using the coaxial technique $\left(\mathrm{LMS}_{\mathrm{c}}\right)$, on the LMS set using the orthogonal technique (LMS $\left.\mathrm{Lrt}\right)$ and on the LMIS of the standard are presented in Table 1.

Table 1. The results of length measurements in the study of two methods for comparing the LMS

\begin{tabular}{|c|c|c|c|}
\hline \multirow{2}{*}{$\begin{array}{c}\text { Measured } \\
\text { length }\end{array}$} & \multicolumn{3}{|c|}{ Measured value, $\mathrm{mm}$} \\
\cline { 2 - 4 } & LMS $_{\mathrm{c}}$ & LMS $_{\text {ort }}$ & Standard LMIS \\
\hline 0 & 0,0577 & 0,0557 & 0,0558 \\
\hline 10 & 10000,2848 & 10000,2841 & 10000,2844 \\
\hline 25 & 25004,5189 & 25004,5178 & 25004,5178 \\
\hline 30 & 30005,2062 & 30005,2069 & 30005,2070 \\
\hline 35 & 35004,0370 & 35004,0370 & 35004,0374 \\
\hline 50 & 50003,3466 & 50003,3439 & 50003,3441 \\
\hline 60 & 60005,3550 & 60005,3545 & 60005,3544 \\
\hline
\end{tabular}

The transmission error of the unit of length was determined from the obtained values of the length of the LMS and LMIS standard according to the formula (1) for each control point. The results of calculating the transmission error of the unit of length in the study of the methods for comparing the LMS and the standard are presented in table 2 and in figure 4.

Table 2. The transmission error of a unit of length in the study of methods for comparing the LMS

\begin{tabular}{|c|c|c|}
\hline \multicolumn{2}{|c|}{ Transmission error of unit of length $(\Delta), \mu \mathrm{m}$} & \multirow{2}{*}{$\begin{array}{c}\text { Measured } \\
\text { length, } \mathrm{m}\end{array}$} \\
\cline { 1 - 2 } Coaxial technique & Orthogonal technique & 0 \\
\hline 1,9 & 0,1 & 10 \\
\hline 0,4 & 0,3 & 25 \\
\hline 1,1 & 0,0 & 30 \\
\hline 0,8 & 0,1 & 35 \\
\hline 0,4 & 0,4 & 50 \\
\hline 2,5 & 0,2 & 60 \\
\hline 0,6 & 0,1 & \\
\hline
\end{tabular}




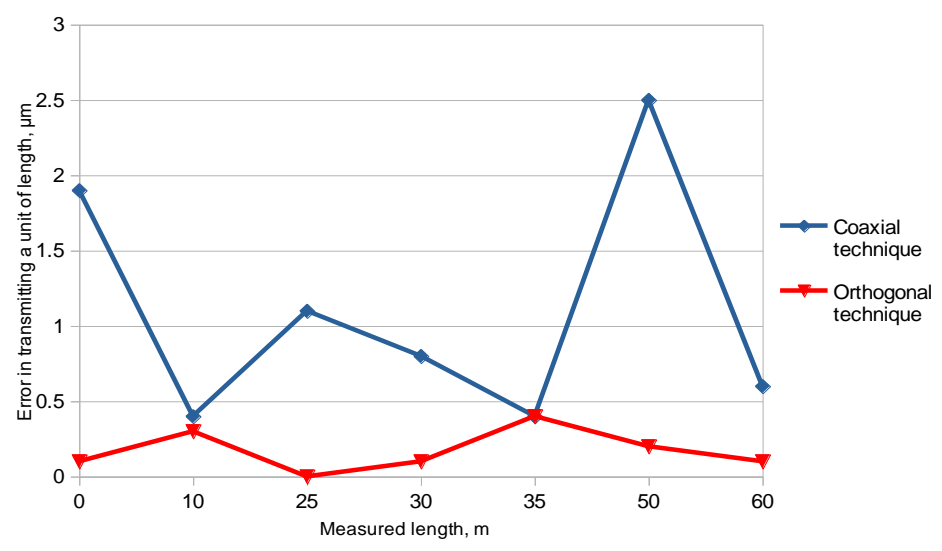

Fig. 4. The graph of the obtained values of the error when measuring by coaxial and orthogonal methods.

As a result of the study, the values of the transmission error of the unit of length were obtained when measuring according to the coaxial and orthogonal methods for comparing the LMS. The transmission error of a unit of length by the coaxial technique is from 0.5 to $2.5 \mu \mathrm{m}$, and by the orthogonal technique from 0.1 to $0.4 \mu \mathrm{m}$. According to the orthogonal technique, the lengths of the optical paths of the laser beams of the LMS and the reference were the same, which reduced the difference in the influence of the non-uniformity of the medium on each laser beam separately, thus reducing the measurement error. Due to the close location of the LMS and the standard, the adjustment of the equipment using the orthogonal technique took half the time compared to the coaxial technique.

\section{Conclusions}

The paper describes the existing method for comparing the LMS. Due to the shortcomings of the coaxial technique, an orthogonal technique for comparing the LMS was proposed. In laboratory conditions, for distances up to $60 \mathrm{~m}$, the values of the distances to the reflector, measured by two LMS, established by the coaxial and orthogonal methods, as well as the LMIS of the standard, were compared, and the results of the study are presented. Orthogonal measurements made it possible to halve the equipment setup time. As a result of the study, when measuring by the orthogonal technique, it was revealed that the error in the transmission of the size of a unit of length was more than twice as large as compared to measurements by the coaxial technique. The use of the orthogonal comparison technique for the LMS calibration will make it possible to carry out measurements with an error of up to $0.5 \mu \mathrm{m}$. 


\section{References}

1. Mise en pratique for the definition of the metre in the SI: SI Brochure, 9th edition, Appendix 22019 URL: https://www.bipm.org/utils/en/pdf/si-mep/SI-App2-metre.pdf (дата обращения 25.09.2021).

2. E.A. Milovanova, K.I. Malikov, N.V. Ivannikova, V.L. Fedorin, N.A. Kononova, K.V. Chekirda, M.Y. Vasil'ev, I.S. Silvestrov, D.A. Sokolov, A.N. Shchipunov, Joint, Measurement Techniques, Investigations of the Metrological Characteristics of the Complex of State Primary Standards of the Unit of Length, T. 61(9), 958, 847(2018)

3. A.G. Sergeev, M.V. Latyshev, V.V. Teregerya, Metrology, standardization, certification: Textbook, 536, 29 (2003)

4. D.A. Sokolov, O.M. Oleinik-Dzyadik, I.S. Silvestrov, Scientific works of the Institute of Astronomy of the Russian Academy of Sciences, Standard measuring complex of length in the range up to $60 \mathrm{~m}$ from the composition of the State primary special standard diameter, no.52, 71, 63 (2020)

5. A.S. Doinikov, Lectures on Metrology, 291, 52 (2018)

6. MI 1214-2000 Recommendation. State system for measuring the interval of measurements. Laser displacement meters. Verification method

7. V. D. Bolshakov, F. Deimlich, A. N. Golubev, V. P. Vasiliev, Radiogeodetic and electro-optical measurements: Textbook for universities. 300, 70 (1985) 